\title{
ON THE CUP PRODUCT FOR GROUPS
}

\author{
M. N. DYER, J. V. LEAHY, AND L. J. MOONEY
}

(Communicated by Frederick R. Cohen)

\begin{abstract}
For $G$ any group and trivial coefficients in $R$, a commutative ring, the authors analyze the kernel of the cup product $\cup: H^{1}(G, R) \otimes H^{1}(G, R) \rightarrow$ $H^{2}(G, R)$ by splicing it together with part of the six-term Hom-Ext exact sequence obtained from $0 \rightarrow I^{2} / I^{3} \rightarrow I / I^{3} \rightarrow I / I^{2} \rightarrow 0$.
\end{abstract}

\section{Statement of Results}

Let $G$ be a group. Let $\mathbb{Z} G$ denote the integral group ring and $I$ the augmentation ideal in $\mathbb{Z} G$, i.e., the kernel of the augmentation map $\varepsilon: \mathbb{Z} G \rightarrow \mathbb{Z}$. The abelian group $\mathbb{Z}$ always has trivial $G$-action. We will constantly identify the first homology group of $G$ with coefficients in $\mathbb{Z}, H_{1} G$, with the quotient $I / I^{2}$. Let $R$ always denote a commutative ring with identity 1 , considered as a trivial $\mathbb{Z} G$-module. If $A$ is an abelian group, we let $A_{R}^{*}=\operatorname{Hom}_{\mathbb{Z}}(A, R)$, considered as an $R$-module via $(r f)(x)=r \cdot f(x) \quad\left(r \in R, f \in A_{R}^{*}, x \in A\right)$.

Definition. Let $R$ be a commutative ring with identity and $A$ a finitely generated abelian group. Let the map $J=J_{A, R}:\left(A_{R}^{*}\right) \otimes_{R}\left(A_{R}^{*}\right) \rightarrow(A \otimes A)_{R}^{*}$ be given by $(J(f \otimes g))(x \otimes y)=f(x) \cdot g(y)$, for $x, y \in A$ and $f, g \in A_{R}^{*}$. The pair $(A, R)$ is effervescent if the map $J_{A, R}$ is an isomorphism. The ring $R$ is effervescent if, for any finitely generated abelian group $A$, the map $J$ is an isomorphism.

Let $\mathbb{Z}_{n}$ denote the integers $\bmod n$ and ${ }_{n} R=\{r \in R \mid n \cdot r=0\}$ be the $n$ torison in $R$. Because $\left(\mathbb{Z}_{n}\right)_{R}^{*} \cong{ }_{n} R$, it is easy to see that $R$ is effervescent iff multiplication induces an isomorphism $\left({ }_{m} R\right) \otimes_{R}\left({ }_{n} R\right) \rightarrow{ }_{m} R$ for any positive integers $m=p^{a}, n=p^{b}$, where $p$ is a prime with exponents $a \leq b$. This leads to

Proposition 1. If $A$ is a finitely generated free abelian group and $R$ is any commutative ring with identity, then the pair $(A, R)$ is effervescent. If $R$ is torsionfree, a finite cyclic group, or a PID, then $R$ is effervescent.

Our first theorem places the cup product in dimension one at the end of the following exact sequence.

Received by the editors August 10, 1990.

1991 Mathematics Subject Classification. Primary 55N45; Secondary 18G15, 55U20. 
Theorem 1. Suppose that $H_{1} G$ is finitely generated as an abelian group and that $R$ is any commutative ring with identity, considered as a trivial $\mathbb{Z} G$-module. Suppose that the pair $\left(H_{1} G, R\right)$ is effervescent. Then the following sequence is exact:

$$
0 \rightarrow\left(I / I^{2}\right)_{R}^{*} \rightarrow\left(I / I^{3}\right)_{R}^{*} \rightarrow H^{1}(G, R) \otimes_{R} H^{1}(G, R) \stackrel{\cup}{\rightarrow} H^{2}(G, R) .
$$

The second map in the sequence is the dual of the projection $I / I^{3} \rightarrow I / I^{2}$, while the third map is essentially the dual of the multiplication $m: I / I^{2} \otimes I / I^{2} \rightarrow I^{2} / I^{3}$ given by taking $[\alpha] \otimes[\beta] \rightarrow[\alpha \beta]$ for $\alpha, \beta \in I$.

The exactness of this sequence (over rational coefficients) was first noticed by making a nontrivial translation of the exact sequence of Chen on the second page of $[\mathrm{C}]$.

The early part of sequence (1.1) is a part of the following (6-term) exact sequence

$$
0 \rightarrow\left(I / I^{2}\right)_{R}^{*} \rightarrow\left(I / I^{3}\right)_{R}^{*} \rightarrow\left(I^{2} / I^{3}\right)_{R}^{*} \stackrel{\partial}{\rightarrow} \operatorname{Ext}_{\mathbb{Z}}\left(H_{1} G, R\right) \rightarrow \cdots
$$

induced by dualizing the exact sequence

$$
0 \rightarrow I^{2} / I^{3} \rightarrow I / I^{3} \rightarrow I / I^{2} \rightarrow 0 .
$$

Furthermore, the exact sequence $0 \rightarrow I^{2} \rightarrow I \rightarrow I / I^{2} \rightarrow 0$ induces a long exact sequence

(1.4)

$$
\begin{array}{ccccccc}
H_{2} G & \stackrel{\kappa}{\rightarrow} & I / I^{2} \otimes I / I^{2} & \stackrel{-m}{\rightarrow} & I^{2} / I^{3} & \rightarrow & 0 \\
\downarrow \partial_{2} \cong & & \cong \uparrow \mathrm{UCT} & & \| & & \\
H_{1}(G, I) & \rightarrow & H_{1}\left(G, I / I^{2}\right) & \underset{\partial}{\rightarrow} & \mathbb{Z} \otimes_{\mathbb{Z} G} I^{2} & \rightarrow & \mathbb{Z} \otimes_{\mathbb{Z} G} I \cong \mathbb{Z} \otimes_{\mathbb{Z} G}\left(I / I^{2}\right)
\end{array}
$$

where UCT is the map from the universal coefficients theorem. The fact that $-\partial=m(\mathrm{UCT})$ is true because one can show that $\partial((a-1) \otimes(b-1))=$ $\left(a^{-1}-1\right)(b-1)+I^{3}$. See the discussion at the end of $\S 2$.

The proof of this theorem involves showing the following lemma, which gives a nice, concrete definition of a part of the cup product in dimension one.

Proposition 2. (a) If $R$ is an arbitrary commutative ring with identity, the following diagram commutes

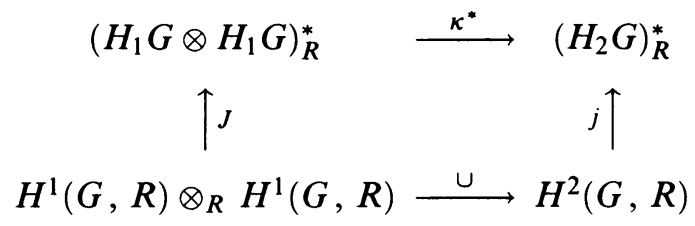

where $j$ is the natural restriction map.

(b) Now assume that the pair $\left(H_{1} G, R\right)$ is effervescent. Let $I \otimes I$ denote the tensor product of the augmentation ideal with itself (tensor over $\mathbb{Z}$, with the diagonal action). Let $\psi: \mathrm{H}_{2} G \rightarrow I / I^{2} \otimes I / I^{2}$ be the homomorphism induced by the reduction $I \otimes I \rightarrow I / I^{2} \otimes I / I^{2}$. Then $\psi^{*}=\kappa^{*}$.

Theorem 2. Assume that $H_{1} G$ is a finitely generated abelian group and that the pair $\left(H_{1} G, R\right)$ is effervescent. Then the following diagram (1.6) is commutative, where $i$ and $j$ are the maps of the universal coefficient theorem. All horizontal 
and vertical sequences are exact. The rightmost vertical sequence is split. The sequence with maps $u, t$, and $\partial$ is also exact, as described above. Specifically, the following maps commute:

(a) $\kappa^{*} J=j \cup$ (Proposition 2),

(b) (U) $J^{-1} m^{*}=i \partial$,

(c) $J s=m^{*} t$ (this defines $s$ ).

The injection $\dot{\kappa}^{*}$ is defined by $\kappa^{*}$ using the exactness of (1.4).

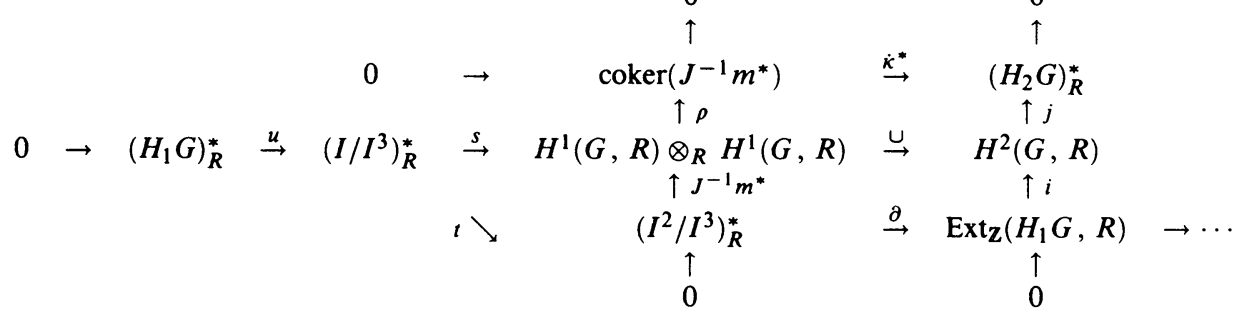

Proof of Theorem 1 from Theorem 2. We need only show that im $s=\operatorname{ker} \cup$. This follows from (a), (b), and (c).

Consider $x \in \operatorname{ker} \cup$. Then $\kappa^{*} J(x)=0$ (by (a)) and so $\left.\exists z \in\left(I^{2} / I^{3}\right)\right)_{R}^{*}$ such that $m^{*}(z)=J(x)$. But by (b), $i \partial(z)=\cup J^{-1} m^{*}(z)=\cup(x)=0$ implies that $\partial(z)=0$. Hence, there is an element $w \in\left(I / I^{3}\right)_{R}^{*}$ so that $t(w)=z$, which yields that $s(w)=x$. Hence $\operatorname{im} s \supseteq \operatorname{ker} \cup$.

For the converse, let $w \in\left(I / I^{3}\right)_{R}^{*}$. Then by (c), Us $(w)=(\cup) J^{-1} m^{*} t(w)=$ $i \partial t(w)=0$. Hence, $\operatorname{im} s=\operatorname{ker} \cup$.

The proofs of these results are given in $\S 2$. A number of corollaries appear in $\S 3$.

\section{Proofs}

For a given presentation of $G: L \rightarrow F(X) \rightarrow G$ ( $X$ is a set of free generators for $F$ and $L$ is the relation subgroup) Brown [B, p. 46] gives a specific isomorphism

$$
\theta^{-1}: F_{2} \cap L /[F, L] \rightarrow H_{2}(G, \mathbb{Z})
$$

where the group on the right-hand side is calculated from the normalized bar resolution [HS, p. 217].

We define $\widetilde{\theta}^{-1}: F_{2} \cap L \rightarrow \mathbb{Z}_{2} G$ and then note that $\tilde{\theta}^{-1}$ passes to the quotients to give the desired map. For $r \in F_{2} \cap L$ then

$$
r=\prod_{j=1}^{q}\left[a_{j}, b_{j}\right]
$$

for some $a_{j}, b_{j} \in F$. For $1 \leq k \leq q$, let $I_{k}=\prod_{j=1}^{k}\left[\dot{a}_{j}, \dot{b}_{j}\right]$ and $\dot{a}$ denote the element in $G$ whose preimage in $F$ is $a$. Then $\tilde{\theta}^{-1}(r)$ is given by the cycle $\left(I_{0}=1\right.$ and $[1 \mid a]=0 \in C_{2} G$ for any $\left.a \in G\right)$

$$
\sum_{1}^{q}\left[I_{i-1} \mid \dot{a}_{i}\right]+\left[I_{i-1} \dot{a}_{i} \mid \dot{b}_{i}\right]-\left[I_{i-1} \dot{a}_{i} \dot{b}_{i} \dot{a}_{i}^{-1} \mid \dot{a}_{i}\right]-\left[I_{i} \mid \dot{b}_{i}\right]
$$


We will give an explicit description of $\kappa$.

By the Brown map $\theta^{-1}$ we know that all elements of $H_{2}(G, \mathbb{Z})$ have a representative of the form $\tilde{\theta}^{-1}(r)$ for some $r \in F_{2} \cap L$, so it is enough to describe it on those elements. The boundary map $\partial_{2}$ of (1.4) is based on the following diagram:

$$
\begin{aligned}
& C_{2} G \otimes_{\mathbb{Z} G} \mathbb{Z} G \\
\downarrow & \rightarrow \partial_{2} \\
C_{1} G \otimes_{\mathbb{Z} G} I \rightarrow C_{2} G \otimes_{\mathbb{Z} G} \mathbb{Z} & \rightarrow C_{1} G \otimes_{\mathbb{Z} G} \mathbb{Z} G
\end{aligned}
$$

We get $\partial_{2}([x \mid y] \otimes 1)=(x[y]-[x y]+[x]) \otimes 1=[y] \otimes x^{-1}-[x y] \otimes 1+[x] \otimes 1$. Using this form we will describe $\partial_{2} \widetilde{\theta}^{-1}(r) \in C_{1} G \otimes_{\mathbb{Z} G} I$.

For $r$ as given above we have

$$
\begin{aligned}
\partial_{2} \tilde{\theta}^{-1}(r)= & \partial_{2}\left(\sum_{1}^{q}\left[I_{i-1} \mid \dot{a}_{i}\right]+\left[I_{i-1} \dot{a}_{i} \mid \dot{b}_{i}\right]-\left[I_{i-1} \dot{a}_{i} \dot{b}_{i} \dot{a}_{i}^{-1} \mid \dot{a}_{i}\right]-\left[I_{i} \mid \dot{b}_{i}\right]\right) \\
= & \sum_{1}^{q}\left(\left[\dot{a}_{i}\right] \otimes\left(I_{i-1}\right)^{-1}-\left[I_{i-1} \dot{a}_{i}\right] \otimes 1+\left[I_{i-1}\right] \otimes 1+\left[\dot{b}_{i}\right] \otimes\left(I_{i-1} \dot{a}_{i}\right)^{-1}\right. \\
& -\left[I_{i-1} \dot{a}_{i} \dot{b}_{i}\right] \otimes 1+\left[I_{i-1} \dot{a}_{i}\right] \otimes 1-\left[\dot{a}_{i}\right] \otimes\left(I_{i-1} \dot{a}_{i} \dot{b}_{i} \dot{a}_{i}^{-1}\right)^{-1}+\left[I_{i-1} \dot{a}_{i} \dot{b}_{i}\right] \otimes 1 \\
& \left.-\left[I_{i-1} \dot{a}_{i} \dot{b}_{i} \dot{a}_{i}^{-1}\right] \otimes 1-\left[\dot{b}_{i}\right] \otimes\left(I_{i}\right)^{-1}+\left[I_{i} \dot{b}_{i}\right] \otimes 1-\left[I_{i}\right] \otimes 1\right) \\
= & \sum_{1}^{q}\left(\left[\dot{a}_{i}\right] \otimes\left(1-\dot{a}_{i} \dot{b}_{i}^{-1} \dot{a}_{i}^{-1}\right) I_{i-1}^{-1}+\left[\dot{b}_{i}\right] \otimes\left(1-\dot{b}_{i} \dot{a}_{i} \dot{b}_{i}^{-1}\right) \dot{a}_{i}^{-1} I_{i-1}^{-1}\right) \\
& +\sum_{1}^{q}\left(\left[I_{i-1}\right] \otimes 1-\left[I_{i}\right] \otimes 1\right)+\sum_{1}^{q}\left(-\left[I_{i-1} \dot{a}_{i} \dot{b}_{i} \dot{a}_{i}^{-1}\right] \otimes 1+\left[I_{i} \dot{b}_{i}\right] \otimes 1\right) .
\end{aligned}
$$

The second and third sums are zero; the third because $I_{i-1} \dot{a}_{i} \dot{b}_{i} \dot{a}_{i}^{-1}=I_{i} \dot{b}_{i}$. Thus

$$
\partial_{2} \tilde{\theta}^{-1}(r)=\sum_{1}^{q}\left(\left[\dot{a}_{i}\right] \otimes\left(1-\dot{a}_{i} \dot{b}_{i}^{-1} \dot{a}_{i}^{-1}\right) I_{i-1}^{-1}+\left[\dot{b}_{i}\right] \otimes\left(1-\dot{b}_{i} \dot{a}_{i} \dot{b}_{i}^{-1}\right) \dot{a}_{i}^{-1} I_{i-1}^{-1}\right)
$$

is a cycle in $C_{1} G \otimes_{Z G} I$.

If we let $\hat{a}$ denote the image of $a \in F$ inside $G_{a b}=H_{1} G$, then we see that

$$
\begin{aligned}
\kappa\left(\theta^{-1}([r])\right)= & \text { class of } \partial_{2} \tilde{\theta}^{-1}(r) \\
= & \left\{\sum _ { 1 } ^ { q } \left(\left[\dot{a}_{i}\right] \otimes\left(1-\dot{a}_{i} \dot{b}_{i}^{-1} \dot{a}_{i}^{-1}\right) I_{i-1}^{-1}\right.\right. \\
& \left.\left.+\left[\dot{b}_{i}\right] \otimes\left(1-\dot{b}_{i} \dot{a}_{i} \dot{b}_{i}^{-1}\right) \dot{a}_{i}^{-1} I_{i-1}^{-1}\right)\right\} \\
= & \sum_{1}^{q}\left(\hat{a}_{i} \otimes \hat{b}_{i}-\hat{b}_{i} \otimes \hat{a}_{i}\right) .
\end{aligned}
$$

It should be noted that the above implies that for $r=\prod_{1 \leq i \leq q}\left[a_{i}, b_{i}\right]^{\alpha_{i}}$ where $\alpha_{i} \in\{-1,1\}$, then $\kappa\left(\theta^{-1}([r])\right)=\sum_{1}^{q} \alpha_{i}\left(\hat{a}_{i} \otimes \hat{b}_{i}-\hat{b}_{i} \otimes \hat{a}_{i}\right)$. 
Lemma 2.1. For any $r \in F_{2} \cap L$ with $r \equiv \prod_{i<j}\left[x_{i}, x_{j}\right]^{\alpha_{i j}}\left(\bmod F_{3}\right)\left(\alpha_{i j} \in \mathbb{Z}\right)$, if $[r]$ represents the class of $r$ in $\mathrm{H}_{2} G$, we have

$$
\kappa([r])=\sum_{i<j} \alpha_{i j}\left(\hat{x}_{i} \otimes \hat{x}_{j}-\hat{x}_{j} \otimes \hat{x}_{i}\right) \in H_{1} G \otimes H_{1} G .
$$

Proof. Observe that $r=\prod_{i<j}\left[x_{i}, x_{j}\right]^{\alpha_{i j}} \cdot \prod_{k=1}^{q}\left[a_{k}, b_{k}\right]$ where $\left[a_{k}, b_{k}\right] \in F_{3}$ for $k=1, \ldots, q$. Since $\left[a_{k}, b_{k}\right] \in F_{3}$ at least one of $\hat{a}_{k}$ or $\hat{b}_{k}$ is zero for each $k$ and hence $\hat{a}_{k} \otimes \hat{b}_{k}=0$. Now use the note preceding this lemma to see

$$
\kappa(r)=\sum_{i<j} \alpha_{i j}\left(\hat{x}_{i} \otimes \hat{x}_{j}-\hat{x}_{j} \otimes \hat{x}_{i}\right)
$$

Definition. $\operatorname{Hom}_{S}(A, B, C)$. If $A \subset B$ is an inclusion of $S$-modules, for some ring $S$ and $C$ is any $S$-module, then we use the notation

$$
\operatorname{Hom}_{S}(A, B, C) \subseteq \operatorname{Hom}_{S}(A, C)
$$

to be the set of $S$-homomorphisms $A \rightarrow C$ that factor through $B$.

Let $K_{2}=\operatorname{ker}\left(\partial_{2}: C_{2} G \rightarrow C_{1} G\right\}$ and let $p: K_{2} \rightarrow C_{2} G$ be the inclusion. Using the fact that $R$ has trivial $\mathbb{Z} G$-action, we compute $H^{2}(G, R)$ as

$$
\begin{aligned}
H^{2}(G, R) & =\operatorname{Hom}_{\mathbb{Z} G}\left(K_{2}, R\right) / \operatorname{Hom}_{\mathbb{Z} G}\left(K_{2}, C_{2} G, R\right) \\
& =\left(\left(K_{2}\right)_{G}\right)_{R}^{*} /\{\text { those homomorphisms that factor through }
\end{aligned}
$$

$$
\left.p_{G}:\left(K_{2}\right)_{G} \rightarrow\left(C_{1}\right)_{G}\right\}
$$

We may identify $\mathrm{H}_{2} G$ as the kernel of $p_{G}$. We will think of the map $j$ : $H^{2}(G, R) \rightarrow\left(H_{2} G\right)_{R}^{*}$ as given by restricting an element of $\left(\left(K_{2}\right)_{G}\right)_{R}^{*}$ to $\left(\operatorname{ker} p_{G}\right)_{R}^{*}$ and then by composing with $\theta$, where $\theta^{-1}: F_{2} \cap L /[F, L] \rightarrow H_{2} G=\operatorname{ker} p_{G}$ was defined above.

Proposition 2.2. Assume that $H_{1} G$ is finitely generated and that $R$ is any commutative ring with identity. Then, in the diagram below, $\kappa^{*} J=j \cup$.

$$
\begin{array}{ccccc}
\left(H_{1} G \otimes H_{1} G\right)_{R}^{*} & \stackrel{\kappa^{*}}{\rightarrow} & \left(H_{2} G\right)_{R}^{*} & = & \left(F_{2} \cap L /[F, L]\right)_{R}^{*} \\
H^{1}(G, R) \otimes_{R} H^{1}(G, R) & \stackrel{\cup}{\rightarrow} & H^{2}(G, R) & = & \left(\left(K_{2}\right)_{G}\right)_{R}^{*} /\left(K_{2}\right)_{G} \rightarrow\left(C_{1}\right)_{G}, \\
& & & \swarrow
\end{array}
$$

where we realize $\kappa: H_{2} G \rightarrow H_{1} G \otimes H_{1} G$ as the composite

$$
F_{2} \cap L /[F, L] \stackrel{\theta^{-1}}{\rightarrow} \operatorname{ker} p_{G} \rightarrow H_{1} G \otimes H_{1} G
$$

given in Lemma 2.1 .

Proof. We write $H_{1} G$ as $\sum_{j=1}^{c} \mathbb{Z}_{\tau_{j}} \oplus \mathbb{Z}^{d}$, where $\tau_{1}>1$ and $\tau_{i}$ divides $\tau_{i+1}$ for $i=1, \ldots, c-1$. Let $u=c+d$ and assume that $\left\{\hat{x}_{1}, \ldots, \hat{x}_{u}\right\}$ is a minimal generating set for $H_{1} G$ with the order of $\hat{x}_{i}=\tau_{i}$ for $i=1, \ldots, c$, and $\left\{\hat{x}_{c+1}, \ldots, \hat{x}_{u}\right\}$ a basis for $\mathbb{Z}^{d}$. Choose the presentation $F$ of $G$ to be in preabelian form, so that we may assume that a subset $\mathfrak{B}=\left\{x_{1}, \ldots, x_{u}\right\}$ of the generators $X$ of $F$ go bijectively onto the chosen generating set for $H_{1} G$. Then $H_{1} G \otimes H_{1} G$ is minimally generated by the set $\left\{\hat{x}_{i} \otimes \hat{x}_{j}: i, j=1, \ldots, u\right\}$. Notice that $\mathbb{Z}_{\tau_{i}} \otimes \mathbb{Z}_{\tau_{j}} \cong \mathbb{Z}_{\min \left\{\tau_{i}, \tau_{j}\right\}}$. Thus, $\left(H_{1} G \otimes H_{1} G\right)_{R}^{*}$ is minimally generated 
(as an $R$-module) by the set of dual maps $\left\{\left(\hat{x}_{i} \otimes \hat{x}_{j}\right)^{*}: i, j=1, \ldots, u\right.$ such that $\left.\left(\hat{x}_{i} \otimes \hat{x}_{j}\right)^{*} \neq 0\right\}$. This set clearly depends exactly on the torsion in $H_{1} G$ and in $R$.

(2.4) Take $r \in F_{2} \cap L$, with $r=\prod_{i<j}\left[x_{i}, x_{j}\right]^{\alpha_{i j}} \cdot s$ where $s \in F_{3}$. We can write $r$ as $r=\prod_{i<j}\left[c_{i j}, d_{i j}\right]^{\left|\alpha_{i j}\right|} \cdot \prod_{1 \leq k \leq q}\left[a_{k}, b_{k}\right]$ where for all $k=1, \ldots, q$, we have $\left[a_{k}, b_{k}\right] \in F_{3}$ and if $\alpha_{i j}>0$ then $c_{i j}=x_{i}$ and $d_{i j}=x_{j}$ and if $\alpha_{i j}<0$ then $c_{i j}=x_{j}$ and $d_{i j}=x_{i}$.

Suppose that $\left(\hat{x}_{n} \otimes \hat{x}_{m}\right)^{*} \neq 0$ and $n<m$. Then, using Lemma 2.1 we see that $\kappa^{*} J\left(\hat{x}_{n}^{*} \otimes \hat{x}_{m}^{*}\right)([r])=\kappa^{*}\left(\hat{x}_{n} \otimes \hat{x}_{m}\right)^{*}([r])=\left(\hat{x}_{n} \otimes \hat{x}_{m}\right)^{*}(\kappa(r))=$ $\left(\hat{x}_{n} \otimes \hat{x}_{m}\right)^{*}\left(\sum_{i<j} \alpha_{i j}\left(\hat{x}_{i} \otimes \hat{x}_{j}-\hat{x}_{j} \otimes \hat{x}_{i}\right)\right)=\alpha_{n m} \cdot 1 \in R$.

But $\left((j \cup)\left[\hat{x}_{n}^{*} \otimes \hat{x}_{m}^{*}\right]\right)([r])=($ class of $)\left(\left(\dot{x}_{n}^{*} \cup \dot{x}_{m}^{*}\right)\left(\theta^{-1}(r)\right)\right)$. We know that for any commutator $\dot{I}$ of $G, \dot{x}_{n}^{*}(\dot{I})=0$, and for $k=1, \ldots, q$ at least one of $\dot{a}_{k}$ or $\dot{b}_{k}$ is in $G_{2}$. Hence $\dot{x}_{n}^{*}\left(\dot{a}_{k}\right) \dot{x}_{m}^{*}\left(\dot{b}_{k}\right)=0$. Using these facts and formula (2.1), we have $\left(\dot{x}_{n}^{*} \cup \dot{x}_{m}^{*}\right)\left(\theta^{-1}(r)\right)=\sum_{i<j}\left|\alpha_{i j}\right|\left(\dot{x}_{n}^{*}\left(c_{i j}\right) \dot{x}_{m}^{*}\left(d_{i j}\right)-\dot{x}_{n}^{*}\left(d_{i j}\right) \dot{x}_{m}^{*}\left(c_{i j}\right)\right)=\alpha_{n m} \cdot 1$. (In order to see the last equality we observe that for $n<m$ and $\alpha_{n m}>0$ then $\left[x_{n}, x_{m}\right]$ appears precisely $\alpha_{n m}$ times as $\left[c_{i j}, d_{i j}\right]$ while $\left[x_{m}, x_{n}\right]$ does not appear; conversely for $\alpha_{n m}<0$ then $\left[x_{m}, x_{n}\right]$ appears $-\alpha_{n m}$ times and $\left[x_{n}, x_{m}\right]$ does not appear.)

By checking in the above formulas and keeping $n<m$, we see that

$$
\kappa^{*} J\left(\hat{x}_{m}^{*} \otimes \hat{x}_{n}^{*}\right)([r])=-\alpha_{n m} \cdot 1=\left((j \cup)\left[\hat{x}_{m}^{*} \otimes \hat{x}_{n}^{*}\right]\right)([r]) .
$$

In case $n=m$ the above shows that both maps evaluated at $\hat{x}_{n}^{*} \otimes \hat{x}_{m}^{*}$ are zero since $\left[x_{n}, x_{n}\right]$ does not appear in any $r$.

We now prove the rest of Proposition 2 ; i.e., that $\Psi^{*}=\kappa^{*}$.

Definition 2.3. Let $I^{(n)}$ denote the tensor product of $I$ with itself (over $\mathbb{Z}$ ) $n$ times, with the diagonal action. Recall from [HS] that the reduced bar construction is given by $C_{n} G=Z G \otimes I^{(n)} \quad(n \geq 0$, and with diagonal action) with the boundary map $\partial_{n}=\varepsilon \otimes 1$. Thus $\operatorname{ker} \partial_{n}=I^{(n+1)}$. Then one computes the second homology of $G$ with coefficients in $\mathbb{Z}$ as

$$
H_{2} G=\left(I \otimes I \cap I \cdot C_{1} G\right) / I(I \otimes I) .
$$

By using the reduced bar resolution $C_{*} G$ in the form of Definition 2.3 we see that $H^{2}(G, R)=\operatorname{Hom}_{\mathbb{Z} G}(I \otimes I, R) / \operatorname{Hom}_{\mathbb{Z} G}\left(I \otimes I, C_{1} G, R\right)$. We will also identify $\operatorname{Hom}_{\mathbb{Z}}\left(I^{2} / I^{3}, R\right)$ with $\operatorname{Hom}_{\mathbb{Z} G}\left(I^{2}, R\right)$.

Consider the reduction map $I \otimes I \rightarrow I / I^{2} \otimes I / I^{2}$. By showing that this map carries $I(I \otimes I)$ to zero, we see that the reduction map induces a homomorphism $\Psi: H_{2}(G, \mathbb{Z}) \rightarrow I / I^{2} \otimes I / I^{2}$.

Proposition 2.4. Let $R$ be any commutative ring. Then the formula $\Psi^{*} J=j \cup$ holds in the following diagram:

$$
\begin{array}{ccc}
\left(H_{1} G \otimes H_{1} G\right)_{R}^{*} & \stackrel{\Psi^{*}}{\rightarrow} & \left(H_{2} G\right)_{R}^{*} \\
\uparrow_{J} & & \uparrow j \\
H^{1}(G, R) \otimes_{R} H^{1}(G, R) & \stackrel{\cup}{\rightarrow} & H^{2}(G, R)
\end{array}
$$

Proof. This is an easy exercise in the definitions of things, using the reduced bar resolution above and the fact that the cup product on the reduced bar construction is just multiplication. 
Corollary 2.5. If the pair $\left(H_{1} G, R\right)$ is effervescent then $\Psi^{*}=\kappa^{*}$.

Proof. This follows because $\Psi^{*}=j \cup J^{-1}=\kappa^{*}$.

We now prove Theorem $2(\mathrm{~b})$.

Proposition 2.6. If the pair $\left(H_{1} G, R\right)$ is effervescent then the following diagram commutes:

$$
\begin{array}{ccc}
H^{1}(G, R) \otimes_{R} H^{1}(G, R) & \stackrel{\cup}{\rightarrow} & H^{2}(G, R) \\
\uparrow J^{-1} & & \uparrow \\
\left(H_{1} G \otimes H_{1} G\right)_{R}^{*} & & \\
\uparrow m^{*} & & \\
\left(I^{2} / I^{3}\right)_{R}^{*} & \stackrel{-\partial}{\rightarrow} & \operatorname{Ext}_{\mathbb{Z}}\left(H_{1} G, R\right)
\end{array}
$$

Proof. In order to compute the Ext functor we use the exact sequence of abelian groups

$$
0 \rightarrow I^{2} \rightarrow I \rightarrow I / I^{2} \rightarrow 0,
$$

so that $\operatorname{Ext}_{\mathbb{Z}}\left(H_{1}(G, \mathbb{Z}), R\right)=\operatorname{Hom}_{\mathbb{Z}}\left(I^{2}, R\right) / \operatorname{Hom}_{\mathbb{Z}}\left(I^{2}, I, R\right)$. Notice that $\left(C_{1} G\right)_{G}=(\mathbb{Z} G \otimes I)_{G}=\mathbb{Z} G \otimes_{\mathbb{Z} G} I=I$. Let $q: I \otimes I \rightarrow C_{1} G$ denote the inclusion. Hence we may identify the image of $q_{G}:(I \otimes I)_{G} \rightarrow\left(C_{1} G\right)_{G}$ as $I^{2}$ and the cokernel as $I / I^{2}$. Notice that under this identification the element $[(x-1) \otimes(y-1)]$ in $(I \otimes I)_{G}$ under $q_{G}$ goes to the element $[(x) \otimes(y-1)]-[1 \otimes(y-1)]$ in $\left(C_{1} G\right)_{G}$. but

$$
\begin{aligned}
{[(x) \otimes(y-1)]-[1 \otimes(y-1)] } & =\left[x\left(1 \otimes x^{-1}(y-1)\right)\right]-[1 \otimes(y-1)] \\
& =\left[1 \otimes x^{-1}(y-1)\right]-[1 \otimes(y-1)] \\
& \rightarrow\left(x^{-1}-1\right)(y-1) \in I^{2} .
\end{aligned}
$$

By using the reduced bar resolution $C_{*} G$ in the form of Definition 2.3 we see that $H^{2}(G, R)=\operatorname{Hom}_{\mathbb{Z} G}(I \otimes I, R) / \operatorname{Hom}_{\mathbb{Z} G}\left(I \otimes I, C_{1} G, R\right)$. We will also identify $\operatorname{Hom}_{\mathbb{Z}}\left(I^{2} / I^{3}, R\right)$ with $\operatorname{Hom}_{\mathbb{Z} G}\left(I^{2}, R\right)$, because $R$ has the trivial action.

Let $\rho: I \otimes I \rightarrow\left(I / I^{2}\right) \otimes\left(I / I^{2}\right)$ be the reduction. First, we define a map

$$
\varphi:\left(H_{1} G \otimes H_{1} G\right)_{R}^{*} \rightarrow H^{2}(G, R)
$$

by $\varphi(\alpha)=$ the class of $\alpha \rho$ in $H^{2}(G, R)$ where $\alpha \in \operatorname{Hom}_{\mathbb{Z} G}\left(\left(I / I^{2}\right) \otimes\left(I / I^{2}\right), R\right)$. Then it is easy to see that

$$
U=\varphi J
$$

We will show that $\varphi m^{*}=i \partial$.

The map $\rho$ induces a map $(I \otimes I)_{G} \rightarrow\left(I / I^{2}\right) \otimes\left(I / I^{2}\right)$ (also called $\rho$ ). The map $q_{G}$ with the new codomain is called $\eta:(I \otimes I)_{G} \rightarrow I^{2}$. Let $\lambda: I^{2} \rightarrow I^{2} / I^{3}$ be the natural projection.

Let $g \in\left(I^{2} / I^{3}\right)^{*}$. Using the presentation (2.7), one may show that $\partial(g)=$ $\{g \lambda\} \in \operatorname{Ext}_{\mathbb{Z}}\left(H_{1}(G, \mathbb{Z}), R\right)$. Now by $(2.8)$, it is enough to show that $g m \rho=$ $-g \lambda \eta$ for any such $g$. But, letting $[(x-1) \otimes(y-1)] \in(I \otimes I)_{G}$ we see that

$$
g m \rho([(x-1) \otimes(y-1)])=g([(x-1)(y-1)])
$$

while

$$
-g \lambda \eta([(x-1) \otimes(y-1)])=-g\left(\left[\left(x^{-1}-1\right)(y-1)\right]\right) .
$$


Now consider $(x-1)\left(x^{-1}-1\right)(y-1) \in I^{3}$ :

$(x-1)\left(x^{-1}-1\right)(y-1)=\left(1-x^{-1}-x+1\right)(y-1)=-\left(x^{-1}-1\right)(y-1)-(x-1)(y-1)$.

Hence, $\left[\left(x^{-1}-1\right)(y-1)\right]=-[(x-1)(y-1)]$ in $I^{2} / I^{3}$. The result follows.

\section{Applications}

The following are corollaries to Theorem 2 .

Corollary 3.1. Let $R=\mathbb{Z}$, the integers. Then the torsionfree part of the cup product is given by the dual of $\kappa$ or $\Psi$. The torsion part of the cup product is $\partial$.

Corollary 3.2. Suppose that $\operatorname{Ext}_{\mathbb{Z}}\left(H_{1} G, R\right)=0$ (e.g., if $R$ is divisible). Then the following sequence is exact:

$$
0 \rightarrow\left(I^{2} / I^{3}\right)_{R}^{*} \stackrel{J^{-1} m^{*}}{\longrightarrow} H^{1}(G, R) \otimes_{R} H^{1}(G, R) \stackrel{\cup}{\rightarrow} H^{2}(G, R) .
$$

Assuming that $R$ is a field of characteristic 0 , we can compare this sequence to that of Hillman $[\mathrm{H}]$ by the natural map $\left(G_{2}\right.$ is the commutator subgroup of $G$ and $\left.G_{3}=\left[G, G_{2}\right]\right)$

$$
\eta: G_{2} / G_{3} \rightarrow I^{2} / I^{3}
$$

given by sending $\{[x, y]\} \rightarrow[(x-1)(y-1)-(y-1)(x-1)]$. Then the following diagram commutes:

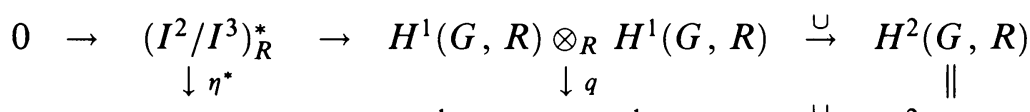

$$
\begin{aligned}
& 0 \rightarrow\left(G_{2} / G_{3}\right)_{R}^{*} \rightarrow H^{1}(G, R) \wedge H^{1}(G, R) \stackrel{\cup}{\rightarrow} H^{2}(G, R) \\
& \begin{array}{ll}
\downarrow & \downarrow \\
0 & 0
\end{array}
\end{aligned}
$$

The map $q$ is the natural surjection from the tensor product to the exterior product. That $\eta^{*}$ is a surjection follows from the commutativity of the diagram.

A group $G$ is a TFT-group if $H_{1} G$ is torsionfree and $H_{2} G$ is a torsion group $[S]$. In this case we see the cup product is trivial.

Corollary 3.3. Let $G$ be a TFT-group with $H_{1} G$ finitely generated. Let $R$ be any commutative ring with identity. Then the cup product $\cup: H^{1}(G, R) \otimes_{R}$ $H^{1}(G, R) \rightarrow H^{2}(G, R)$ is trivial.

Proof. First, we observe that because $H_{1} G$ is torsionfree and finitely generated, it is finitely generated free abelian. Hence, the pair $\left(H_{1} G, R\right)$ is effervescent for any ring $R$. By the lemma on page 150 of [S] (or by just staring at sequence (1.4) and observing that the hypotheses show that $\kappa=0$ ), the multiplication map $m: H_{1} G \otimes H_{1} G \rightarrow I^{2} / I^{3}$ is an isomorphism. It follows that $J^{-1} m^{*}$ is an isomorphism. Thus the cup product is concentrated in the lower part of diagram (1.6). But $H_{1} G$ is free abelian, hence $\operatorname{Ext}_{\mathbb{Z}}\left(H_{1} G, R\right)=0$. Therefore, the cup product vanishes.

Let $\rho(A)$ denote the rank of the finitely generated abelian group $A$ and $\Delta_{i}=\rho\left(I^{i} / I^{i+1}\right) \quad(i \geq 1)$. Then we have 
Corollary 3.4. Let $R=\mathbb{Z}$, the integers. Then $\rho(\mathrm{im} \cup)=\Delta_{1}^{2}-\Delta_{2}=\rho(\operatorname{im} \kappa)$.

A similar formula occurred in [D], in conjunction with a natural filtration in the homology of groups.

We now give an application of (1.1) to CW-complexes.

Corollary 3.5. Let $X$ be a connected $C W$-complex with fundamental group $G$. Suppose that $H_{1} X=H_{1} G$ is finitely generated and that $\left(H_{1} G, R\right)$ is an effervescent pair. Then the following sequence is exact.

$$
0 \rightarrow H^{1}(X, R) \rightarrow\left(I / I^{3}\right)_{R}^{*} \rightarrow H^{1}(X, R) \otimes_{R} H^{1}(X, R) \stackrel{\cup}{\rightarrow} H^{2}(X, R) .
$$

Proof. Because $X$ is a topological space we have, by Hopf's theorem [B, p. 41], a natural surjection from $\mathrm{H}_{2} \mathrm{X} \rightarrow \mathrm{H}_{2} G$. Thus, for any $R$, there is a natural injection from $H^{2}(G, R) \rightarrow H^{2}(X, R)$. The result then follows from (1.1) and the naturality of the cup product.

\section{REFERENCES}

[B] K. Brown, Cohomology of groups, Graduate Texts in Math., vol. 87, Springer-Verlag, New York, 1982.

[C] K.-T. Chen, Algebras of iterated path integrals and fundamental groups, Trans. Amer. Math. Soc. 156 (1971), 359-379.

[D] M. Dyer, Cofiltrations in the cohomology of groups, manuscript, 1988.

[HS] P. Hilton and U. Stammbach, A course in homological algebra, Graduate Texts in Math., vol. 4, Springer-Verlag, New York, 1971.

[H] J. Hillman, The kernel of the cup product, Bull. Austral. Math. Soc. 32 (1985), 261-274.

$\left[\mathrm{H}_{1}\right] \quad$ - The kernel of integral cup product, J. Austral. Math. Soc. Ser. A 42 (1987).

[S] R. Strebel, A note on groups with torsion-free abelianization and trivial multiplicator, Comment. Math. Helv. 54 (1979), 147-158.

[W] J. Wood, A theorem on the injectivity of the cup product, Proc. Amer. Math. Soc. 37 (1973), 301-304.

Department of Mathematics, University of Oregon, Eugene, Oregon 97403 